Review

\title{
Dietary Needs, Approaches and Recommendations to Meet the Demands of Future Manned Space Flights
}

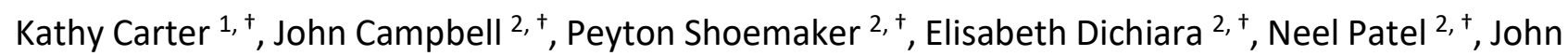
Caruso $^{2,+}{ }^{*}$

1. Central State University, 1400 Brush Row Rd, Wilberforce OH, USA; E-Mail: kcarter2@centralstate.edu

2. University of Louisville, 2100 S Floyd St, Louisville KY, USA; E-Mails: john.campbell@louisville.edu; peyton.shoemaker@louisville.edu; elisabeth.dichiara@louisville.edu; neel.patel@louisville.edu; ifcaru01@louisville.edu

$\dagger$ These authors contributed equally to this work.

* Correspondence: John Caruso; E-Mail: jfcaru01@louisville.edu

Academic Editor: Jennifer Keogh

Special Issue: Feature Papers of Recent Progress in Nutrition 2021

Recent Progress in Nutrition

2022, volume 2, issue 1

doi:10.21926/rpn.2201005
Received: December 09, 2021

Accepted: February 20, 2022

Published: February 23, 2022

\begin{abstract}
Space flight imposes a myriad of adverse effects upon the human body. Improved in-flight nutritional strategies help mitigate those adverse effects. This review examines how space flight changes human physiology, and how improved in-flight nutritional strategies may address the unique dietary needs created by microgravity exposure. Dietary approaches and recommendations to address those needs are discussed. This review also describes food sustainability methods for long-term manned space flight, how precision nutrition can aid inflight dietary prescriptions, and how wearable technologies can help monitor dietary status and health during long-term manned space flight. This review describes the magnitude of adverse changes incurred from space flight, and the current knowledge base on how improved in-flight nutritional strategies may abate the adverse changes. Wearable
\end{abstract}

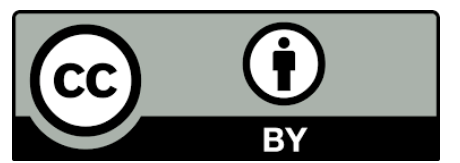

(c) 2022 by the author. This is an open access article distributed under the conditions of the Creative Commons by Attribution License, which permits unrestricted use, distribution, and reproduction in any medium or format, provided the original work is correctly cited. 
technologies, used in conjunction with precision nutrition prescriptions, may best serve the demands of monitoring human health and dietary status on future manned space flights.

\section{Keywords}

Negative energy balance; microgravity; food sustainability; precision nutrition; wearable technology

\section{Introduction}

We performed a literature review on approaches to address dietary needs to meet the demands space flight imposes upon humans. This review includes and references material from original studies, which all received appropriate Institutional Review Board approval and were carried out ethically in accordance with Declaration of Helsinki guidelines. Articles comprising this paper include those pertaining to the physiological demands and dietary needs of microgravity, food supply sustainability, precision nutrition and technologies with the potential to monitor in-flight nutritional status. Our approach to this review was to include articles from each of these broad areas that were also applicable to manned space flight. From our initial literature review, we acquired over 500 articles, technical reports, book chapters and web site urls. We searched MedLine and Scorpus databases to acquire those 500 items. With exclusion criteria that included the terms "space flight", "microgravity", and "bed rest", we narrowed the list to the 56 references that comprise this novel literature review. We believe the 56 are those most related and pertinent to space flight nutrition among the 500 we initially acquired.

Space flight is an environment that is both unique and inhospitable to human life [1, 2]. Yet despite the hazards and challenges it poses, manned flights continue, as NASA has announced goals of landing a woman on the moon by 2024, developing a sustainable human lunar presence by 2028 , and taking humans to Mars in the 2030s [3,4]. Nutrition is but one in-flight countermeasure to the many adverse effects incurred by astronauts, who must cope with this novel environment as they perform mission objectives [4-6]. All body systems change in response to space flight. Nutrition seeks to abate such adverse effects that include, but are not limited to, higher metabolic rates/inefficient body heat removal, altered cardiovascular and immune system function, increased radiation exposure, and mass and strength losses to weight-bearing muscles and bones. We also discuss strategies to provide food for long-term missions, monitor in-flight food intakes, and how precision nutrition and wearable technologies may ensure individual crewmember's dietary recommendations are met during space flights.

\section{Adverse Effects of Space Flight}

\subsection{Higher Metabolic Rates/Inefficient Body Heat Removal}

Upon acute microgravity exposure, body systems comprised of large fluid volumes perhaps undergo the most rapid and dramatic changes, which in turn have pronounced effects on metabolism, hydration, and excess heat removal. The removal of body heat is vital to human life, yet in space it does not occur as easily as on Earth. Metabolic rates are elevated in space in large 
part due to the higher energy costs required to remove excess heat $[7,8]$. To compensate, humans expend more energy in microgravity to accomplish this task $[1,9,10]$. As a result, all types of inflight physical activity have an additive effect on heat production and inevitably elicit higher energy costs than comparable tasks done on Earth. Energy costs in microgravity can be quite high. For instance, human energy costs ranged from 500-1320 kcal $\cdot \mathrm{hr}^{-1}$ for extravehicular activities [11-13]. Rapid and dramatic body fluid shifts in space also impair evaporative heat loss. Blunted thirst sensations and lower fluid intakes compound this problem [13, 14]. In-flight fluid shifts centralize blood volumes, as a result the body expends more energy to pump blood to the skin surface to enable evaporation. Lower fluid intakes evoke dehydration and cause perspiration rates to decline with longer missions, while sweating in space creates a film over the skin that impairs convective heat loss $[10,15]$. Thus, astronauts often return to Earth from longer flights with $1-5 \%$ body and muscle mass losses, hyperthermic and dehydrated [6, 10, 14-17]. Better in-flight nutritional and hydration strategies must be devised to abate these effects.

Taste and smell loss that reduces food palatability [4], limited food preparation time [18-21], and menu fatigue contribute to body and muscle mass losses. In-flight energy needs are estimated from World Health Organization (WHO) equations and Dietary Reference Intake values [16]. Energy guidelines for flights up to 360 days include protein intakes of $0.8 \mathrm{~g} \cdot \mathrm{kg}^{-1}$ body mass, $350 \mathrm{~g} \cdot \mathrm{day}^{-1}$ of carbohydrates, and $70 \mathrm{~g} \cdot \mathrm{day}^{-1}$ of fat, which equate to $12-15 \%$ of total kcals from protein, $50-55 \%$ total kcals from carbohydrate, and 30-35\% total kcals from fat $[4,9,19,20]$. Assuming a pre-flight body mass of $90 \mathrm{~kg}$ and a daily intake of $2318 \mathrm{kcals}$, this equates to ranges of 278-348 kcals for protein, 1159-1275 kcals for carbohydrate, and 695-811 kcals of fat. Early in-flight energy intakes were typically $30-40 \%$ below WHO guidelines, which create negative energy balances and impact electrolyte balance, gut microflora and metabolism $[4,8,11,18]$. To offset such changes, in-flight nutrition requires higher fluid and energy intakes. To address this issue, starting with NASA-Mir flights of the late 1990s, the U.S. and Russia agreed to nutrition and food quality standards, which included taste and palatability assessments before launch, and menus planned with four meals per 24 hours [22]. Since then, energy intakes were maintained at $85-95 \%$ of predicted requirements [23]. While adequate energy is perhaps the most important aspect of in-flight nutrition, it is unknown if higher energy and protein intakes have a muscle-sparing effect independent of exercise $[20,24,25]$.

Nutritional concerns also extend to post-flight health. Zwart et al. stated "Data from 11 International Space Station (ISS) astronauts suggest nutritional status is compromised after longduration space flight [26]. Low energy intake is a crucial issue for crewmembers since it is also associated with inadequate vitamin and mineral intake." For instance, pre-launch astronaut red blood cell and folate levels were measured near the upper limit of their normal range, but postflight drop to the lower limit of their normal range [2]. It is believed inadequate food intake, rather than diets low in folate, is the cause of these losses [2]. Hematocrit losses also occur [13, 25], yet blood composition changes little due to in-flight dehydration.

\subsection{Altered Cardiovascular and Immune System Function}

In-flight body fluid redistribution and dehydration also have rapid and adverse effects on cardiovascular and immune system function [27, 28]. Microgravity limits venous pooling and redistributes fluids to the head and torso [2]. When combined with low in-flight vascular resistance, 
hemodynamics in space function in stark contrast to the manner done on Earth. While the cardiovascular system adapts quickly to space, astronauts exhibit their poorest aerobic capacity upon return to Earth, when they incur large $\mathrm{VO}_{2}$ max losses despite performance of in-flight steady state exercise [2]. Dehydration leads to drops in plasma volume and aerobic capacity [28]. To compensate, heart rates rise during and after missions [28, 29].

The immune system is at the forefront of the body's ability to cope with physical and mental stressors imposed by space flight $[25,28,30]$. Altered immune cell function in space may evoke unresolved inflammation that impacts cognition, mood, and behaviour $[25,28,30]$. In turn, astronaut health and in-flight operational objectives are impaired by such changes $[25,30]$. In microgravity, immune system perturbations are also impacted by nutrition and exercise, each of which affects the body's ability to function [30,31]. Early nutritional research looking at supplements of zinc, dietary fat, antioxidants, glutamine and carbohydrate to improve immune function saw little value [32]. However, the immune system may benefit from higher $\Omega-3$ fatty acid, $\beta$-alanine and carnosine intakes [33]. $\Omega$-3 fatty acids, exert alterations on the activation of cell of the immune system; as part of the cellular membrane if affects the fluidity of the membrane, and recently has been indicated as a signalling molecule within the immune system [34]. It has also been noted that carnosine supplementation helps regulate immune function through regulating functions of neutrophils [35]. Recent work implied diets with the standard NASA in-flight macronutrient ratio lack adequate amounts of these nutrients, and was concluded immune system function may benefit from in-flight supplementation [33]. Given the benefits of $\Omega-3$ fatty acid supplementation [33], continued research should assess if $\Omega-6$ fatty acid intakes should also increase to comply with the fatty acid ratio cited by the NASA Constellation Program. New research on in-flight $\beta$-alanine and carnosine supplementation is warranted, which may alter the list of required nutrients for long-term flights [36].

\subsection{Increased Radiation Exposure}

Manned space flights expose astronauts to higher radiation [37, 38]. Effective in-flight ionizing radiation doses vary from $50-2,000 \mathrm{mSv}$, which are primarily a function of mission duration, where $1 \mathrm{mSv}$ equals the radiation from three chest $\mathrm{x}$-rays [38]. Even low radiation doses compromise numerous enzymes and intestinal transporters [4]. Beyond low Earth orbit, space radiation increases crewmember's risk of cancer, radiation sickness, central nervous system ailments and other degenerative diseases [37, 38]. Sadly, one or more of these disorders could potentially develop on future long-term missions, even among crewmembers with no pre-flight predisposition or family history for these ailments [37, 38]. Proper in-flight nutrition appears vital to abate the occurrence of these ailments. Vitamin $\mathrm{C}$ decreases cataract risk formation, which may occur from radiation exposure over time. It also protects against nucleated bone marrow cell loss in mice exposed to sublethal irradiation levels [39]. Little information exists on the effect of nutrient protection in neurons from radiation. Garlic extracts and polyphenolics from fruits and vegetables may assist in preventing, or at least slowing down, radiation damage to the nervous system [39]. Serum vitamin B, D, E and $K$ levels, as well as superoxide dismutase, are essential to cope with greater radiation. Unfortunately, all decline in microgravity $[2,4]$. Higher antioxidant levels limit the severity of in-flight oxidative stress [25]. 


\subsection{Mass and Strength Losses to Weight-bearing Muscles and Bones}

Omission of normal weight-bearing and ambulatory activity evokes musculoskeletal mass and strength loss. To illustrate the adverse effects microgravity has on musculoskeletal loss, postmission muscle strength losses that exceed $10 \%$ of preflight values were deemed operationally significant and may impair emergency egress performance; sadly, such losses can occur within the first 40 days of non-weight bearing activity $[24,40]$. Strength losses are in part due to increases in whole-body protein turnover. Protein synthesis is increased; however, protein degradation occurs at greater rates [20]. Most protein decreases occur during the first month of flight, yet the rate of loss does not appear to increase with longer space flights [22].

While human bone remodels over 4-6 month cycles, the absence of mechanical loading hastens skeletal losses [41]. The magnitude of bone loss is a function of mission length, skeletal (cortical vs. trabecular) composition and its degree of weight-bearing responsibility [41]. Such losses are accompanied by greater bone resorption. Unlike most body changes, full bone mass and density restoration does not occur when astronauts return to Earth, which makes these losses perhaps the most dangerous. Bone mineral density loss is among the most important health issues, and specific nutrients must be provided to limit this effect. Originally, ISS food was high in sodium which can increase bone loss [20]. Sodium has since been reduced within in-flight food. Vitamins D and $K$ metabolism and function are impaired with long flights, which also undermines bone health [2]. Calcium and phosphate supplements did not abate bone resorption, yet $\Omega$ - 3 fatty acid intakes were correlated to less bone loss [4].

\section{Strategies to Address In-flight Food Demands}

\subsection{Food Supplies for Long-term Manned Space Missions}

Sustainable food supplies for long-term missions are essential if nutritional countermeasures are to abate microgravity's adverse effects and preserve astronaut health and performance. A balance among multiple factors may offer the best sustainability and nutritional approach for space flight [21]. Numerous solutions have been proposed to address food supply sustainability; currently NASA's pre-packaged foods have a stated shelf life of about two years, yet a trip to Mars will require five years of stored room temperature pre-packaged food, thereafter nutrient depletion occurs [42, 43]. Soil simulations are but one proposed solution to sustainable food supplies. Research on soil simulations revealed the Mars environment, with its $\mathrm{CO}_{2}$-rich atmosphere and soil rich in minerals, was superior to that of the Moon for growing plants [43]. It was proposed the surface of Venus may also be suitable for agriculture [43]. Research is ongoing to determine how the atmosphere and weather patterns will affect plant growth. Another proposed solution, to combat radiation and atmospheric challenges posed by inhabiting other planets, involves a closed soilless cultivation system. This solution aims to mimic Earth's soil conditions and composition and is perhaps a viable option for planets chosen for long-term inhabitation and/or colonization. Hydro- and aquaponics were suggested as a solution when a viable water supply is available. Finally, aeroponics allows crops to be planted and harvested year-round without interruptions or contaminations [43]. More research is needed to determine how best to supply food to space travellers over extended time periods. 
To provide in-flight foodstuffs to crewmembers, the safety, nutrition, and acceptability of an implemented food system must be carefully weighed against use of space craft resources that include, but are not limited to, it's energy supply and time allocated to food preparation [44, 45]. Multiple factors undermine the quality of space food freshness and quality over time, they include processing and storage [45]. Vitamins D and $\mathrm{K}$, as well as potassium and calcium, may be quite susceptible to processing and storage issues, as these nutrients are offered in limited quantities in space-flown food [45]. Others suggest current space technology does not allow food preservation for five years, and advocate for retort sterilized pouches $[45,46]$. Use of the pouches provided promising results; meat- and vegetable-based items lasted 2-8 years without refrigeration when stored within the pouches. Vitamin B and C losses occurred in foods stored within the pouches, however most foods maintained nutritional value throughout their shelf life [46]. Given the challenges of storing and delivering foods to astronauts to provide a sustainable supply of food for long-term manned space flights, retort sterilized pouches deserve continued inquiry to address this issue [46]. Future food sustainability research suggests space agencies should examine all potential solutions, as well as be amenable to other future options.

\subsection{Precision Nutrition and Tracking In-flight Food Intakes}

Monitoring astronauts' nutritional status is important to ensure crewmember health is preserved as spaceflight durations increase [2]. Dietary intakes have been tracked with various methods. For early flights of short duration, foods were weighed by dieticians prior to flights. Food masses returned to Earth were subtracted from the food loaded pre-flight and differences were analyzed for macro and micronutrient intakes. Barcode readers were used and placed on individual food items. Scanned package labels noted how much of an item was eaten. The calculation of nutrient intakes occurred post flight. Another method saw ISS crewmembers complete weekly food questionnaires and data were downloaded on Earth for analysis to enable dietary changes to occur in real time [18]. Questionnaires were decidedly more accurate for nutrient intake determinations since in-flight menus have been typically quite limited.

Nutrition is important to good health. The extreme environment of microgravity alters the way the body processes nutrients. Research shows individual biological responses may differ to the same foods when they are eaten [46]. Precision nutrition, a new area of research under the umbrella concept of precision medicine, develops individualized methods to prevent or treat chronic diseases based on a person's unique characteristics such as DNA, race, gender, health history, and lifestyle [5]. Exposure to microgravity can exacerbate these differences and make precision nutrition vital to in-flight diet prescription. Precision nutrition may thus offer the best nutrition-based countermeasure to the adverse effects of microgravity. Precision Nutrition, which utilizes information from metabolomics and nutrigenomics, has the potential to provide individualized nutrient intake recommendations based on how an individuals' metabolic pathways are potentially modified by their diet [47]. For example, Huang et al., found that individuals with a lower genetic risk of diabetes benefitted from a low-protein diet, while individuals with a higher genetic risk had improved insulin resistance and $\beta$ cell function with a high protein diet [48]. While Precision Nutrition research is still in its infancy it may prove vital to the prescription of in-flight diets for space flight crewmembers. With the small, yet increasingly diverse, number of people exposed to 
microgravity, precision nutrition may serve as a valid approach to abate the dietary imbalances incurred from long-term space flights [22].

The idea for in-flight precision nutrition in part comes from data provided by astronauts who underwent long-duration flights aboard the ISS [23]. In addition to blood and urine sample obtained before and after space flights, the astronauts provided self-assessments of their diets, in the form of food frequency questionnaires, administered at the completion of 6-10 day in-flight menu cycles aboard the ISS [23]. Results included significant iron, hematocrit, calcium, vitamin D and folate losses $[23,42]$. Parathyroid hormone, which also declines with space flight, impacts both vitamin $D$ and calcium metabolism; deficits in all three contribute to post-flight bone mineral density losses incurred by the weight-bearing skeleton [49]. Advancements in precision nutrition now include dietary supplementation both in-flight and during bed rest (a microgravity analog) to abate such losses. However, it is important to remember the concept of in-flight precision nutrition is in its infancy and not without constraints and limitations. Among them are logistical issues when anything is to be implemented aboard manned space flights, as well as tailoring diets to the momentary desires of crewmembers throughout long-term missions and having foods present to meet those desires. Furthermore, current evidence that shows precision nutrition is better and more costeffective than traditional food preparation approaches is limited [47]. Future research should balance genomics, metabolomics and nutritional needs of individual astronauts with current inflight constraints and limitations to best devise personalized in-flight dietary approaches to space flight's deleterious effects [25].

\subsection{Precision Nutrition and Wearable/Hand-held Technologies}

In addition to specific in-flight dietary deficiencies that precision nutrition can address, it must also attend to negative energy balances, an issue visited upon human physiology upon immediate exposure to microgravity [7]. In conjunction with future research, precision nutrition prescriptions also seek to tailor and enhance in-flight diets for individual astronauts through technology advancements. Such technologies include wearable and hand-held devices that easily assess health [50-53], metabolism and nutrition status [54]. Three such devices monitor health and metabolism status through the body's waste products, which in turn offer insights on the nutritional needs of individuals [51-53]. One device utilizes stool recognition artificial intelligence technology to identify individual dietary needs [51]. The device uses neural networks to quantify changes to a person's stool samples over time [51]. Another device, small and handheld, measures hydration, electrolytes, $\mathrm{pH}$, vitamin $\mathrm{C}$, uric acid and ketones from 1-2 urine drops placed onto a test strip inserted with its reader [52]. The device uses spectrometry and reportedly offers accurate and reliable measurements [52]. Finally, a third device assesses metabolism with respiratory gas sensors and flow meters from a single breath [53]. The small handheld device instantly quantifies a person's respiratory quotient and reportedly underwent multiple validation studies [53]. Other technologies entail non-invasive devices to assess nutritional status, as well as hormonal, enzymatic and immune function, from saliva $[50,54]$. Such technologies capitalize on the on-going advancements and sensor miniaturization $[50,54]$.

Given the importance of these measurements to astronauts, particularly when data are collected in-flight, they offer unique insights into crewmember health and their nutritional needs. Furthermore, given the limited area aboard the ISS and other space-related vehicles, miniaturized 
devices appear to be ideal for use aboard future manned flights. The information such technologies provide can accurately tailor in-flight precision nutrition prescriptions. For instance, wearables may one day assess $\Omega-3$ fatty acid, $\beta$-alanine and carnosine intakes, which improve cognitive function and immune health, yet are deficient in isocaloric diets administered at the in-flight macronutrient (55\% carbohydrate, $30 \%$ fat, $15 \%$ protein) ratio [33]. With isocaloric diets prescribed for two weeks, daily $\Omega-3$ fatty acid, $\beta$-alanine and carnosine intakes (mean \pm sem) were $0.35 \pm 0.07 \mathrm{~g}, 371.3 \pm 62.5$ $\mathrm{mg}$ and $6729.6 \pm 8.3 \mu \mathrm{mol} \cdot \mathrm{mg}^{-1}$ respectively [33]. $\Omega-3$ fatty acid intakes were far less than those required for long-term missions, yet no recommended daily allowance (RDA) for $\beta$-alanine and carnosine exists [36]. Given Baba et al.'s results, higher $\Omega-3$ fatty acid intakes than those listed by Cooper et al. may be prudent and best achieved, given the challenges of in-flight nutrition, by supplementation $[33,36]$. Continued research may wish to examine if $\Omega-6$ fatty acid intakes should also increase to comply with the fatty acid ratio approved for the NASA Constellation Program, as well as if in-flight $\beta$-alanine and carnosine supplementation should be considered, which may alter the list of required nutrients for long-term space missions [36].

\section{Conclusions}

Given the negative energy balances produced by microgravity, and the additive effect extravehicular activities have upon metabolic rates [11,12,17], NASA is incorporating wearable technologies to assess the in-flight operational performance of astronauts as their health is monitored $[55,56]$. Wearables currently include continuous heart rate measurements that offer indirect evidence of in-flight stress levels experienced by astronauts, as well as when, and to what extent, metabolic rates rise during space missions $[55,56]$. In combination with data from emerging sensor-based technologies [50-54] in-flight information on astronaut health and nutritional needs can be sent in real time to NASA personnel on Earth. Wearable technologies, used in conjunction with precision nutrition prescriptions, may best serve the demands of monitoring astronaut health and dietary status on future manned space flights.

\section{Author Contributions}

Kathy Carter and John Caruso conceptualized and wrote this review article. John Campbell, Peyton Shoemaker, Elisabeth Dichiara and Neel Patel each assisted the aforementioned authors in acquiring, reading and approving papers that comprise this review article's reference section.

\section{Competing Interests}

The authors have declared that no competing interests exist.

\section{References}

1. Grassi B. Bed rest studies as analogs of conditions encountered in space and in diseases. Med Sci Sports Exerc. 2018; 50: 1907-1908.

2. Mulavara AP, Peters BT, Miller CA, Kofman IS, Reschke MF, Taylor LC, et al. Physiological and functional alterations after spaceflight and bed rest. Med Sci Sports Exerc. 2018; 50: 1961-1980.

3. Dumbar B. Human needs: Sustaining life during exploration [Internet]. Washington: NASA; 2007. Available from: https://.nasa.gov/vision/earth/everydaylife/jamestown-needs-fs.html. 
4. Cahill T, Hardiman G. Nutritional challenges and countermeasures for space travel. Nutr Bull. 2020; 45: 98-105.

5. Schmidt MA, Goodwin TJ. Personalized medicine in human space flight: Using omics based analyses to develop individualized countermeasures that enhance astronaut safety and performance. Metabolomics. 2013; 9: 1134-1156.

6. Smith SM, Zwart SR, Block G, Rice BL, Davis-Street JE. The nutritional status of astronauts is altered after long-term space flight aboard the International Space Station. J Nutr. 2005; 135: 437-443.

7. Bergouignan A, Stein TP, Habold C, Coxam V, O'gorman D, Blanc S. Towards human exploration of space: The Theseus review series on nutrition and metabolism research priorities. NPJ Microgravity. 2016; 2: 16029.

8. Stein TP. The relationship between dietary intake, exercise, energy balance and the space craft environment. Pflugers Arch. 2000; 441: R21-R31.

9. Lane HW, Schoeller DA. Nutrition in spaceflight and weightlessness models. Boca Raton: CRC Press; 1999.

10. Leach CS, Leonard JI, Rambaut PC, Johnson PC. Evaporative water loss in man in a gravity-free environment. J Appl Physiol. 1978; 45: 430-436.

11. Grigoryev A, Bugrov SA, Bogomolov VV, Egorov AD, Kozlovskaya I, Pestov I, et al. Review of primary medical results of year-long flight on Mir station. Kosm Biol Aviakosm Med. 1990; 24: 3-10.

12. Johnston RS, Dietlein LF. Biomedical results from Skylab. Washington: Scientific and Technical Information Office, National Aeronautics and Space Administration; 1977.

13. Wickman L, Nota B, Keates S. Lunar life support system study: Metabolic energy and water considerations [Internet]. Reston: American Institute of Aeronautics and Astronautics; 2004. Available from: https://static.apu.edu/static/src/sites/researchscience/downloads/cris lunar life.pdf.

14. Sato $M$, Kanikowska D, Iwase $S$, Shimizu $Y$, Inukai $Y$, Nishimura $N$, et al. Effects of encouraged water drinking on thermoregulatory responses after 20 days of head-down bed rest in humans. Int J Biometeorol. 2009; 53: 443-449.

15. Leach CS, Rambaut PC. Endocrine responses in long-duration manned space flight. Acta Astronaut. 1975; 2: 115-127.

16. Di Girolamo FG, Biolo G, Fiotti N, Situlin R, Piacenza C, Lepore P, et al. The Nutriss study: A new approach to calibrate diet and exercise in long-term space missions to maintain body fat, muscle and fluid homeostasis. Aerotec Missili Spaz. 2020; 99: 121-125.

17. Stein TP, Leskiw MJ, Schluter MD, Hoyt RW, Lane HW, Gretebeck RE, et al. Energy expenditure and balance during spaceflight on the space shuttle. Am J Physiol Regul Integr Comp Physiol. 1999; 276: R1739-R1748.

18. Douglas GL, Zwart SR, Smith SM. Space food for thought: Challenges and considerations for food and nutrition on exploration missions. J Nutr. 2020; 150: 2242-2244.

19. Heer M, Titze J, Smith SM, Baecker N. Nutrition physiology and metabolism in spaceflight and analog studies. Berlin: Springer International Publishing; 2015.

20. Lane HW, Bourland C, Barrett A, Heer M, Smith SM. The role of nutritional research in the success of human space flight. Adv Nutr. 2013; 4: 521-523. 
21. Lane HW, Gretebeck RJ, Schoeller DA, Davis-Street J, Socki RA, Gibson EK. Comparison of ground-based and space flight energy expenditure and water turnover in middle-aged healthy male US astronauts. Am J Clin Nutr. 1997; 65: 4-12.

22. Lane HW, Feeback DL. History of nutrition in space flight: Overview. Nutrition. 2002; 18: 797804.

23. Smith SM, Zwart SR. Nutrition issues for space exploration. Acta Astronaut. 2008; 63: 609-613.

24. Caruso JF, Hamill JL, Yamauchi M, Mercado DR, Cook TD, Higginson B, et al. Albuterol augments resistance exercise to attenuate ankle extensor strength loss. J Appl Physiol. 2005; 98: 17051711.

25. Enrico C. Space nutrition: The key role of nutrition in human space flight. arXiv. 2016. arXiv: 1610.00703.

26. Zwart SR, Mathews Oliver SA, Fesperman JV, Kala G, Krauhs J, Ericson K, et al. Nutritional status assessment before, during, and after long-duration head-down bed rest. Aviat Space Environ Med. 2009; 80: A15-A22.

27. Chuong $P$, Wysoczynski $M$, Hellmann J. Do changes in innate immunity underlie the cardiovascular benefits of exercise? Front Cardiovasc Med. 2019; 6: 70.

28. Lee S, Feiveson AH, Stein S, Stenger MB, Platts SH. Orthostatic intolerance after ISS and space shuttle missions. Aerosp Med Hum Perform. 2015; 86: A54-A67.

29. Loehr JA, Guilliams ME, Petersen N, Hirsch N, Kawashima S, Ohshima H. Physical training for long-duration spaceflight. Aerosp Med Hum Perform. 2015; 86: A14-A23.

30. Gleeson M, Nieman DC, Pedersen BK. Exercise, nutrition and immune function. J Sports Sci. 2004; 22: 115-125.

31. Crucian BE, Choukèr A, Simpson RJ, Mehta S, Marshall G, Smith SM, et al. Immune system dysregulation during spaceflight: Potential countermeasures for deep space exploration missions. Front Immunol. 2018; 9: 1437.

32. Nieman DC. Exercise immunology: Nutritional countermeasures. Can J Appl Physiol. 2001; 26: S45-S55.

33. Baba S, Smith T, Hellmann J, Bhatnagar A, Carter K, Vanhoover A, et al. Space flight diet-induced deficiency and response to gravity-free resistive exercise. Nutrients. 2020; 12: 2400.

34. Gutiérrez S, Svahn SL, Johansson ME. Effects of omega-3 fatty acids on immune cells. Int J Mol Sci. 2019; 20: 5028.

35. Li YF, He RR, Tsoi B, Li XD, Li WX, Abe K, et al. Anti-stress effects of carnosine on restraint-evoked immunocompromise in mice through spleen lymphocyte number maintenance. PloS One. 2012; 7: e33190.

36. Cooper M, Douglas G, Perchonok M. Developing the NASA food system for long-duration missions. J Food Sci. 2011; 76: R40-R48.

37. Chancellor JC, Scott GB, Sutton JP. Space radiation: The number one risk to astronaut health beyond low earth orbit. Life. 2014; 4: 491-510.

38. Perez J. Why space radiation matters [Internet]. Washington: NASA; 2009 [cited date 2021 March 5th]. Available from: https://www.nasa.gov/analogs/nsrl/why-space-radiation-matters.

39. Turner ND, Braby LA, Ford J, Lupton JR. Opportunities for nutritional amelioration of radiationinduced cellular damage. Nutrition. 2002; 18: 904-912.

40. Adams GR, Hather BM, Dudley GA. Effect of short-term unweighting on human skeletal muscle strength and size. Aviat Space Environ Med. 1994; 65: 1116-1121. 
41. Collet PH, Uebelhart D, Vico L, Moro L, Hartmann D, Roth M, et al. Effects of 1-and 6-month spaceflight on bone mass and biochemistry in two humans. Bone. 1997; 20: 547-551.

42. Douglas GL, Wheeler RM, Fritsche RF. Sustaining astronauts: Resource limitations, technology needs, and parallels between spaceflight food systems and those on Earth. Sustainability. 2021; 13: 9424.

43. Oluwafemi FA, De La Torre A, Afolayan EM, Olalekan-Ajayi BM, Dhital B, Mora-Almanza JG, et al. Space food and nutrition in a long term manned mission. Adv Astronaut Sci Technol. 2018; 1: 1-21.

44. Catauro PM, Perchonok MH. Assessment of the long-term stability of retort pouch foods to support extended duration spaceflight. J Food Sci. 2012; 77: S29-S39.

45. Cooper M, Perchonok M, Douglas GL. Initial assessment of the nutritional quality of the space food system over three years of ambient storage. NPJ Microgravity. 2017; 3: 17.

46. Rodgers GP, Collins FS. Precision nutrition-the answer to "what to eat to stay healthy". JAMA. 2020; 324: 735-736.

47. Wang DD, Hu FB. Precision nutrition for prevention and management of type 2 diabetes. Lancet Diabetes Endocrinol. 2018; 6: 416-426.

48. Huang $T$, Ley $S H$, Zheng $Y$, Wang $T$, Bray GA, Sacks FM, et al. Genetic susceptibility to diabetes and long-term improvement of insulin resistance and $\beta$ cell function during weight loss: The preventing overweight using novel dietary strategies (pounds lost) trial. Am J Clin Nutr. 2016; 104: 198-204.

49. Smith SM, Heer M. Calcium and bone metabolism during space flight. Nutrition. 2002; 18: 849852.

50. Salimetrics. Homepage [Internet]. Carlsbad: Salimetrics, LLC.; 2022 [cited date 2021 December 1st]. Available from: https://salimetrics.com.

51. Auggi. Homepage [Internet]. New Yrok: augGI Technologies Inc.; 2021 [cited date 2021 December 1st]. Available from: https://www.auggi.ai/.

52. Bisu. Homepage [Internet]. Tokyo: Bisu, Inc.; 2021 [cited date 2021 December 1st]. Available from: https://www.bisu.com/home/.

53. Lumen. Homepage [Internet]. Tel Aviv: MetaFlow Ltd.; 2022 [cited date 2021 December 1st]. Available from: https://www.lumen.me/.

54. UC San Diego. New wearable sensor tracks Vitamin C levels in sweat [Internet]. La Jolla: UC San Diego; 2020 [cited date 2021 December 1st]. Available from:

https://www.ucsdnews.ucsd.edu/pressrelease/VitaminC wearable.

55. NASA. Remote monitoring promotes community health beyond hospitals [Internet]. Washington: NASA; 2020 [cited date 2021 December 1st]. Available from: https://spinoff.nasa.gov/Spinoff2020/hm 1.html.

56. NASA. Biometric sensor tracks vital signs for health [Internet]. Washington: NASA; 2019 [cited date 2021 December 1st]. Available from: https://spinoff.nasa.gov/Spinoff2019/hm 6.html. 


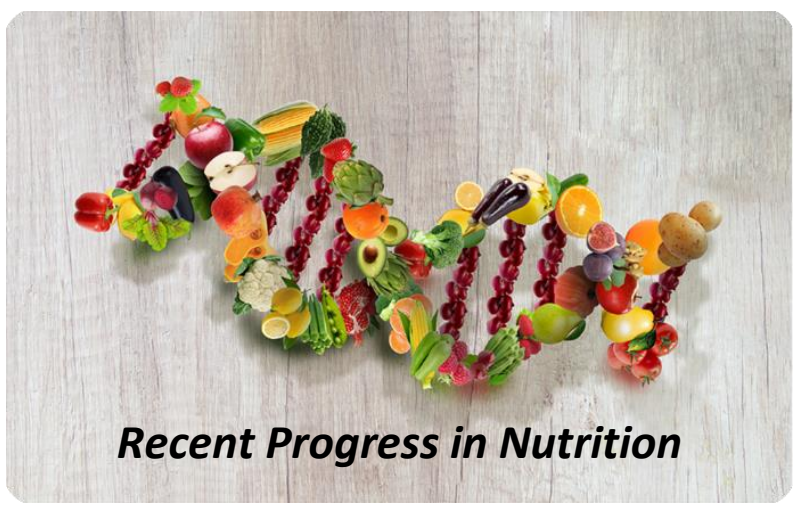

Enjoy Recent Progress in Nutrition by:

1. Submitting a manuscript

2. Joining in volunteer reviewer bank

3. Joining Editorial Board

4. Guest editing a special issue

For more details, please visit:

http://www.lidsen.com/journals/rpn 